\title{
ERRATUM: "PHOTOIONIZATION MODELING OF OXYGEN K ABSORPTION IN THE INTERSTELLAR MEDIUM, THE CHANDRA GRATING SPECTRA OF XTE J1817-330” (2013, ApJ, 768, 60)
}

\author{
E. Gatuzz ${ }^{1}$, J. García ${ }^{2,3}$, C. Mendoza ${ }^{1,4}$, T. R. Kallman ${ }^{3}$, M. WitthoefT ${ }^{3}$, A. LohfinK ${ }^{2}$, \\ M. A. Bautista ${ }^{5}$, P. Palmeri ${ }^{6}$, And P. Quinet ${ }^{6,7}$ \\ ${ }^{1}$ Centro de Física, Instituto Venezolano de Investigaciones Científicas (IVIC), P.O. Box 20632, Caracas 1020A, Venezuela; \\ egatuzz@ivic.gob.ve, claudio@ivic.gob.ve \\ ${ }^{2}$ Department of Astronomy and Maryland Astronomy Center for Theory and Computation, University of Maryland, College Park, MD 20742, USA; \\ javier@astro.umd.edu, alohfink@astro.umd.edu \\ ${ }^{3}$ NASA Goddard Space Flight Center, Greenbelt, MD 20771, USA; timothy.r.kallman@ nasa.gov, michael.c.witthoeft@ nasa.gov \\ ${ }^{4}$ Centro Nacional de Cálculo Científico Universidad de Los Andes (CeCalCULA), Corporación Parque Tecnológico de Mérida, Mérida 5101, Venezuela \\ ${ }^{5}$ Department of Physics, Western Michigan University, Kalamazoo, MI 49008, USA; manuel.bautista@ wmich.edu \\ ${ }^{6}$ Astrophysique et Spectroscopie, Université de Mons-UMONS, B-7000 Mons, Belgium; palmeri@umons.ac.be, quinet@umons.ac.be \\ ${ }^{7}$ IPNAS, Sart Tilman B15, Université de Liège, B-4000 Liège, Belgium \\ Received 2013 August 19; published 2013 November 6
}

In the published version of this paper, there are some minor inaccuracies in the absorption-line wavelengths listed in Table 4 as a result of a faulty reduction procedure of the Obs6615 spectrum. The shifts have been detected in a comparison with the wavelengths listed for this spectrum in the Chandra Transmission Grating Catalog and Archive (TGCat ${ }^{8}$ ). They are due to incorrect centroid positions of the zero-order image in both reductions as determined by the tgdetect utility which, when disentangled, yield the improved line positions of the amended Table 4 given below. It must also be pointed out that other quantitative findings of the original paper:

1. Table 5, p. 9: the column density $\left(N_{\mathrm{H}}\right)$, ionization parameter, oxygen abundance of the warmabs model and the normalization and photon index of the power-law model;

2. Table 6, p. 9: the hydrogen column density of the warmabs fit;

3. Table 7, p. 9: the present oxygen equivalent widths of XTE J1817-330; and

4. Table 8, p. 10: the present oxygen column densities of XTE J1817-330 derived from both the curve of growth and warmabs model fit

have been revised in the new light and are, within the estimated uncertainty ranges, in good accord with the new rendering.

Table 4

Absorption Line Assignments

\begin{tabular}{|c|c|c|c|}
\hline Ion & Transition & $\begin{array}{l}\lambda^{\mathrm{a}} \\
(\AA)\end{array}$ & $\begin{array}{l}\lambda^{\mathrm{b}} \\
(\AA)\end{array}$ \\
\hline$\overline{\mathrm{OVII}}$ & $1 s^{2}{ }^{1} S-[1 s] 2 p{ }^{1} P^{\mathrm{o}}$ & $21.593 \pm 0.002$ & $21.60 \pm 0.06$ \\
\hline O vI & $2 s^{2} S-[1 s] 2 s 2 p^{2} P^{\mathrm{o}}$ & $22.024 \pm 0.003$ & \\
\hline O II & $2 p^{3}{ }^{4} S^{\mathrm{o}}-[1 s] 2 p^{3} 4 p^{4} P$ & $22.108 \pm 0.004$ & \\
\hline O II & $2 p^{3}{ }^{4} S^{\mathrm{o}}-[1 s] 2 p^{3} 3 p^{4} P$ & $22.287 \pm 0.004$ & \\
\hline O I & $2 p^{4}{ }^{3} P-[1 s] 2 p^{4}\left({ }^{2} P\right) 4 p^{3} P^{\mathrm{o}}$ & $22.612 \pm 0.006$ & \\
\hline OI & $2 p^{4}{ }^{3} P-[1 s] 2 p^{4}\left({ }^{2} P\right) 3 p^{3} P^{\mathrm{o}}$ & $22.692 \pm 0.005$ & \\
\hline OI & $2 p^{4}{ }^{3} P-[1 s] 2 p^{4}\left({ }^{4} P\right) 4 p^{3} P^{\mathrm{o}}$ & $22.794 \pm 0.002$ & \\
\hline OI & $2 p^{4}{ }^{3} P-[1 s] 2 p^{4}\left({ }^{4} P\right) 3 p^{3} P^{\mathrm{o}}$ & $22.889 \pm 0.004$ & $22.91 \pm 0.03$ \\
\hline O III & $2 p^{2}{ }^{3} P-[1 s] 2 p^{3}{ }^{3} P^{\mathrm{o}}$ & $23.057 \pm 0.003$ & $23.13 \pm 0.09$ \\
\hline O III & $2 p^{2}{ }^{3} P-[1 s] 2 p^{3}{ }^{3} D^{\mathrm{o}},{ }^{3} S^{\mathrm{o}}$ & $23.110 \pm 0.004$ & $23.13 \pm 0.09$ \\
\hline O II & $2 p^{3}{ }^{4} S^{\mathrm{o}}-[1 s] 2 p^{4}{ }^{4} P$ & $23.346 \pm 0.002$ & $23.35 \pm 0.03$ \\
\hline OI & $2 p^{43} P-[1 s] 2 p^{53} P^{\mathrm{o}}$ & $23.506 \pm 0.001$ & $23.52 \pm 0.02$ \\
\hline
\end{tabular}

Notes.

a Present work.

${ }^{b}$ Sala et al. (2007).

We thank members of the Chandra team for useful discussions relating the data processing, especially Michael Nowak for many valuable suggestions.

\section{REFERENCE}

Sala, G., Greiner, J., Bottacini, E., \& Haberl, F. 2007, Ap\&SS, 309, 315

8 http://tgcat.mit.edu/ 